\title{
ÍNDICE DE DESAGREGAÇÃO DO SOLO BASEADO EM ENERGIA ULTRA-SÔNICA(1)
}

\author{
M. A. CAROLINO de SÁ(2), J . M. LIMA(3), \\ M. L. N.SILVA ${ }^{(4)}$ \& M. S. DIAS J UNIOR ${ }^{(5)}$
}

\begin{abstract}
RESUMO
A estabilidade de agregados tem sido determinada por meio de peneiramento úmido, impacto de gotas e, mais recentemente, sonificação. 0 objetivo deste trabalho foi propor um índice baseado em níveis de energia ultra-sônica para expressar a estabilidade de agregados, utilizando material de horizontes A e B de Latossolo R oxo e Terra Roxa Estruturada. Agregados de 4,76 a 7,93 mm foram submetidos a níveis crescentes de energia ultra-sônica. 0 índice de dispersão (massa de agregados secos e, ou, partículas $<0,053 \mathrm{~mm} / \mathrm{massa}$ inicial de agregados) foi plotado em função da energia correspondente para dispersão de cada amostra. As curvas de dispersão foram linearizadas, dividindo-se a energia pelo índice de dispersão, obtendo-se uma equação do tipo EA/ID $=a+b E A$. 0 índice de desagregação foi obtido, dividindo-se os termos b/a da equação, tendo como unidade $\mathrm{mL} \mathrm{J}^{-1}$. Quanto menor o índice de desagregação, maior a estabilidade de agregados, principalmente para amostras dos horizontes $A$ de ambos os solos. A vantagem desse índice sobre um único nível de energia ou mesmo o diâmetro médio geométrico se deve ao fato de ele considerar toda a faixa de energia empregada na curva de dispersão.
\end{abstract}

Termos de indexação: ultra-som, Latossolo Roxo, Terra Roxa Estruturada, agregação.

\footnotetext{
(1) Parte da Tese de Mestrado apresentada pelo primeiro autor à Universidade F ederal de Lavras - UF LA. Recebido para publicação em maio de 1998 e aprovado em abril de 1999.

(2) Engenheiro-Agronômo, M.Sc., UFLA. Caixa Postal 37, CEP 37200-000 Lavras (MG).

(3) Engenheiro-Agronômo, Ph.D., Professor do Departamento de Ciência do Solo, UFLA. Bolsista do CNPq.

(4) Engenheiro-Agronômo, D.Sc., Professor do Departamento de Ciência do Solo, UFLA. Bolsista do CNPq.

(5) Engenheiro-Agrícola, Ph.D., Professor do Departamento de Ciência do Solo, UFLA. Bolsista do CNPq.
} 


\title{
SUMMARY: AGGREGATE STABILITY INDEX BASED ON SONICATION ENERGY
}

\begin{abstract}
Aggregate stability has been measured by wet sieving, water dropping and sonication. The objective of this study is to propose an index (energy - level based index) to express aggregate stability in A and B horizon aggregates of Dusky Red Latosol and Structured Dusky Red Earth. Aggregates from 4.76 to $7.93 \mathrm{~mm}$ weresubmitted to sonication at increasing energy level s. A dispersion ratio $(<0.053 \mathrm{~mm}$ aggregate + particl efraction/ total aggregate mass) was plotted against energy levels, for each sample. These dispersion curves were linearized dividing energy level by dispersion ratioto givean $E A / I D=a+b E A$ typeequation. A dispersion index was obtained dividing $\underline{b}$ by $\underline{a}$, from the equation. Thehigher theaggregate stability, the lower the dispersion index, mainly on A horizon samples of both soils. The advantage of this index over the use of a single energy level or even the geometric mean diameter is that it uses a whol erange of energy levels used for the dispersion curve.
\end{abstract}

Index terms: ultrasound, dusky Red Latosol, structured dusky Red Earth, aggregation.

\section{INTRODUÇÃO}

A susceptibilidade dos agregados do solo à quebra pode ter um efeito significativo em propriedades de interesse agronômico, tais como distribuição do tamanho de poros, condutividade hidráulica, susceptibilidade à compactação e erosão (Raine \& So, 1993).

A formação e a estabilidade de agregados no solo estão relacionadas basicamente com a espessura e constituição da dupla camada elétrica e da composição mineralógica (Veiga et al., 1993; I gwe et al., 1995), matéria orgânica (Tisdall \& Oades, 1982; Roth et al., 1991; Faccin, 1995; Fuller et al., 1995; Campos et al., 1995), tipo de uso agrícola (Fuller et al., 1995) e sistema de cultivo (Sidiras et al., 1984; Eltz et al., 1989; Roth et al., 1991; Derpsch et al., 1991; Campos et al., 1995).

A argila dispersa pelo ultra-som é uma medida adequada para avaliar a quebra de agregados, (Fuller \& Goh, 1992). Segundo Gregorich et al. (1988), a argila dispersa, a baixos níveis de energia, provém de material que liga microagregados entre si, formando macroagregados (diâmetro maior que $0,25 \mathrm{~mm}$ ), enquanto microagregados somente são destruídos a altos níveis deenergia, concordando com Eriksen et al. (1995).

Considerando que a estabilidade de macroagregados é influenciada pelo manejo do solo (Tisdall \& Oades, 1982), baixos níveis de energia ultra-sônica representam melhor o potencial de destruição de agregados pelo manejo, conforme observado por North (1979). Portanto, um índice de estabilidade de agregados que emprega altos níveis de energia, levando à dispersão total das partículas do solo, não é adequado para estudos com objetivo de relacionar o efeito do manejo sobre a estrutura do solo.
North (1976) propôs uma metodologia para quantificar a energia gasta na dispersão de agregados por meio de ultra-som. Entretanto, Koenigs (1978) encontrou falhas metodológicas na maneira decalcular a energia eno uso de dispersante químico. Foi somente com o trabal ho de Raine \& So (1993) que a metodologia proposta por North foi aperfei çoada, tornando possível o cál culo da energia efetivamente gasta para dispersão dos agregados.

Outra maneira mais simples de relacionar energia ultra-sônica com dispersão foi proposta por Christensen (1985). Consisteem expressar a energia emitida pelo aparelho por unidade de volume de suspensão sol o-água, sendo esse critério adotado em diversos trabal hos (Christensen \& Sorensen, 1985; Christensen, 1985, 1986, 1987; Gregorich et al., 1989; Fuller \& Goh, 1992; Fuller et al., 1995; Eriksen et al., 1995).

Revisões de literatura sobre dispersão ultrasônica indicam que não há um método padrão em uso (Watson, 1971; Christensen, 1985). Numerosos fatores, como potência liberada pel o aparel ho, tempo de sonificação, relação solo-água, especificações do equipamento, temperatura da suspensão, gás dissolvido e profundidade de inserção da haste do aparel ho na suspensão, podem influir na eficiência da dispersão (E dwards \& Bremner, 1967; Saly, 1967; North, 1976; Raine \& So, 1994). Entretanto, é bastante promissor o uso de ultra-som em estudos de agregação do sol o, uma vez que a mensuração da energia aplicada a uma suspensão constitui uma base conveniente para comparação entre trabal hos que relacionam a quebra de agregados de solos sob diferentes condições (Raine \& So, 1993).

O objetivo deste estudo foi propor um índice de desagregação, considerando diferentes níveis de energia ultra-sônica em horizontes $A$ e $B$ de Latossolo Roxo e Terra Roxa Estruturada. 


\section{MATE RIAL E MÉTODOS}

Foram utilizados materiais de horizontes $A$ e $B$ de Latossolo Roxo (LR) e Terra Roxa Estruturada (TR), coletados no município de Lavras (MG), localizado a $21^{\circ} 13^{\prime} \mathrm{S}$ e $44^{\circ} 57^{\prime} \mathrm{W}$ Gr. O primeiro foi amostrado no campus da Universidade Federal de Lavras, em terço superior de encosta, sob vegetação natural (floresta tropical subperenifólia), sendo o horizonte A coletado de 0 a $20 \mathrm{~cm}$ e o horizonte B col etado de 80 a $100 \mathrm{~cm}$. A Terra Roxa Estruturada foi amostrada em terço médio de encosta, sob pastagem de grama batatais (Paspalum notatum), com presença de arbustos, sendo o horizonte $\mathrm{A}$ col etado de 0 a $20 \mathrm{~cm}$ e o horizonte $B$ de 40 a $70 \mathrm{~cm}$.

Para caracterização mineralógica da fração argila, foram medidos os teores de caulinita e gibbsita, por meio de análise termo-diferencial (ATD) e identificados os minerais por meio de difratometria de raios-X (Klug \& Alexander, 1974), para complementação das informações a respeito da composição da fração argila.

Os teores de cálcio trocável e carbono orgânico das amostras foram determinados, segundo EMBRAPA (1979).

A densidade de partículas $\left(D_{p}\right)$ foi avaliada pelo método do picnômetro, segundo Blake \& Hartge (1986a), e a densidade do solo $\left(D_{s}\right)$ pelo método do torrão parafinado, segundo Blake\& Hartge, (1986b). A granulometria do sol ofoi determinada pel o método da pipeta (Day, 1965), empregando-se $\mathrm{NaOH} 1 \mathrm{~N}$ como dispersante químico e agitação rápida (6.000 rpm) durante $20 \mathrm{~min}$. Os valores de argila total e argila dispersa em água foram obtidos pela análise granulométrica, segundo metodologia da EMBRAPA (1979).

Para sonificação das amostras, utilizou-se um aparel ho marca Misonix, modelo XL2020TM, operando a $20 \mathrm{kHz}$ no estádio 9, fornecendo uma potência de 105,90 $\pm 1,72 \mathrm{~W}$, cal culada pel o método descrito em Carolino de Sá (1998). Cada amostra (quatro repetições) correspondeu a $10 \mathrm{~g}$ de agregados (peso seco em estufa a $105^{\circ} \mathrm{C}$ ) com tamanho entre 4,76 e 7,93 mm. Antes da sonificação, os agregados foram submetidos a um pré-umedecimento por capilaridade durante duas horas, conforme Marques (1996), que consistiu em colocá-l os em uma bandeja com areia úmida coberta por uma fol ha de papel de filtro. A sonificação foi feita em béquer com $400 \mathrm{~mL}$ de água destilada (dando uma relação solo:água destilada de 1:40), durante 5, 15, 30,60, 300, 900, 1.800 e $2.700 \mathrm{~s}$, o que corresponde às energias aplicadas (EA) de 1,32; 3,97; 7,94; 15,88; 79,42; 238,27; 476,53 e 714,80 J mL-1, expressas, segundo Christensen (1985), por meio da equação 1.

$$
E A=\left[P_{C} \cdot t_{s}\right] / v
$$

em que
EA é a energia aplicada à suspensão ( $\left.\mathrm{mL}^{-1}\right)$,

$P_{c}$ éa potência emitida pelo aparel ho, obtida por meio de calibração (W),

$t_{s}$ é o tempo de sonificação (s) e

$v$ é o volume da supensão $(\mathrm{mL})$.

A ponta da haste do aparelho, de dimensões $175 \mathrm{~mm}$ de comprimento por $19 \mathrm{~mm}$ de diâmetro, foi introduzida na suspensão a uma profundidade de $85 \mathrm{~mm}$. Para reduzir o efeito da alta temperatura, a amostra foi colocada em banho de gelo durante a sonificação, ficando a temperatura da suspensão abaixo de $40^{\circ} \mathrm{C}$, conforme sugerido por Gregorich et al. (1989).

Após cada sonificação, as amostras foram cuidadosamente passadas em peneira de 0,053 mm, quantificando-se a fração dispersa (silte + argila) por diferença (massa inicial deagregados menos a fração retida na peneira, ambos massa seca em estufa a $\left.105^{\circ} \mathrm{C}\right)$. F oi então cal culado o índice de dispersão ID $=[($ fração $<0,053 \mathrm{~mm}) /($ massa inicial de agregados secos)] para cada tempo de sonificação, sendo os resultados normalizados para os teores desilte+argila das amostras e pl otados em curvas de dispersão. Os índices não normalizados foram utilizados na comparação com a agitação mecânica. As curvas foram linearizadas e ajustadas matematicamente. A linearização foi feita, plotando-se, no eixo das abscissas, o nível de energia aplicado à suspensão (EA) em J $\mathrm{mL}^{-1} \mathrm{e}$, no eixo das ordenadas, esse mesmo nível de energia dividido pelo índice de dispersão normalizado (EA/ID), ajustando-se uma equação do tipo y $=a+b x$ para cada amostra por meio de regressão. O termo b/a, cuja unidade é $\mathrm{mL} \mathrm{J}^{-1}$, foi usado como um índice para expressar a suscepti bilidade dosol oà desagregação, uma vez que representa o comportamento da curva que considera todos os níveis de energia aplicados.

Assim, o model o ajustado foi uma equação do tipo $y=[x /(a+b x)]$, sendo y o índice de dispersão (ID), $x$ a energia aplicada à amostra (EA), em J $\mathrm{mL}^{-1}$, ea e b os coeficientes da equação.

\section{RESULTADOS E DISCUSSÃO}

Os índices de dispersão obtidos pela análise granulométrica, empregando-se dispersante químico, estão geralmente abaixo dos índices máximos não normalizados obtidos pelo ultra-som (Quadro 1), mostrando ser a energia ultra-sônica a $714,8 \mathrm{~J} \mathrm{~mL}-1$ mais eficiente para dispersão do que a agitação mecânica, concordando com Saly (1967), mesmo sem o uso de dispersante químico (E dwards \& Bremner, 1967; Genrich \& Bremner, 1972). O horizonte A do Latossolo Roxo (Figura 1) apresentou agregados mais estáveis, atingindo valores constantes deI $D$ normalizados em níveis de energia 
Quadro 1. Distribuição de partículas e, ou, agregados para horizontes A e B do Latossolo Roxo e da Terra Roxa Estruturada

\begin{tabular}{|c|c|c|c|c|c|}
\hline Solo & ID(1) & $I D^{(2)}$ & Argila & Silte & Areia \\
\hline & \multicolumn{5}{|c|}{$-\mathrm{g} \mathrm{kg}^{-1}$} \\
\hline & \multicolumn{5}{|c|}{ Com dispersante NaOH $1 \mathrm{~mol} \mathrm{~L}^{-1}$} \\
\hline LR A & - & 0,83 & 710 & 120 & 170 \\
\hline LR B & - & 0,86 & 670 & 190 & 140 \\
\hline $\mathrm{TRA}$ & - & 0,76 & 440 & 320 & 240 \\
\hline \multirow[t]{2}{*}{ TR B } & - & 0,89 & 700 & 190 & 110 \\
\hline & \multicolumn{5}{|c|}{ Sem dispersante } \\
\hline LR A & 0,89 & 0,19 & 90 & 100 & 810 \\
\hline LR B & 0,90 & 0,22 & 110 & 110 & 780 \\
\hline TR A & 0,88 & 0,49 & 190 & 300 & 510 \\
\hline TR B & 0,93 & 0,73 & 400 & 330 & 270 \\
\hline
\end{tabular}

(1) ID = índice de dispersão não normalizado obtido por ultrasom a 714,8 J mL ${ }^{-1}$. (2) ID =índice de dispersão obtido em análise granulométrica. acima de 476,53 $\mathrm{mL}^{-1}$, enquanto o horizonte $A$ da Terra Roxa e o horizonte B do Latossolo requereram menor energia para a dispersão total, cerca de 238,27 J mL-1. O horizonte B da Terra Roxa Estruturada apresentou agregados menos estáveis, necessitando apenas de 79,42 J mL-1 para dispersão total por ultra-som. Entretanto, vale ressaltar que, neste trabalho, o que está sendo chamado de dispersão total por ultra-som corresponde ao nível de energia acima do qual o índice de dispersão (ID) se estabiliza, podendo ainda existir flóculos ou agregados do tamanho de silte que passaram pela peneira, não sendo portanto quantificados. Como observado por Gregorich et al. (1988), microagregados estáveis só são efetivamente destruídos após aplicados $1.500 \mathrm{~J} \mathrm{~mL}^{-1}$, ou seja, um nível de energia muito acima do máximo utilizado nestetrabal ho que foi de $714,80 \mathrm{~J} \mathrm{~mL}^{-1}$.

As curvas de dispersão linearizadas encontramse na figura 2, onde se observam el evados valores de $\mathrm{R}^{2}$ para todos os casos. Uma vez que os model os

Quadro 2. Características químicas, mineralógicas e físicas dos horizontes A e B do Latossolo Roxo e da Terra Roxa Estruturada

\begin{tabular}{|c|c|c|c|c|c|c|c|c|}
\hline Solo & $\begin{array}{l}\text { Carbono } \\
\text { orgânico }\end{array}$ & Cálcio & Gibbsita & Caolinita & $\mathbf{G b} /(\mathbf{G b}+\mathbf{C t})$ & $D_{p}{ }^{(1)}$ & $D_{s}^{(2)}$ & $\begin{array}{l}\text { Volume total } \\
\text { de poros }\end{array}$ \\
\hline & $\mathrm{g} \mathrm{kg}^{-1}$ & $\mathrm{mmol}_{\mathrm{c}} \mathrm{dm}^{-3}$ & \multicolumn{2}{|c|}{$\mathrm{g} \mathrm{kg}^{-1}$} & & \multicolumn{2}{|c|}{$-\mathrm{kg} \mathrm{dm}^{-3}$} & $\mathrm{~m}^{3} \mathrm{~m}^{-3}$ \\
\hline LR A & 35 & 5 & - & - & - & 2,70 & 1,24 & 0,5392 \\
\hline LR B & 15 & 6 & 383 & 351 & 0,52 & 2,85 & 1,26 & 0,5564 \\
\hline TR A & 18 & 50 & - & - & - & 2,90 & 1,55 & 0,4658 \\
\hline TR B & 7 & 40 & 36 & 363 & 0,09 & 2,96 & 1,67 & 0,4364 \\
\hline
\end{tabular}

${ }^{(1)} D_{p}=$ Densidade de partículas. ${ }^{(2)} D_{s}=$ Densidade do solo.

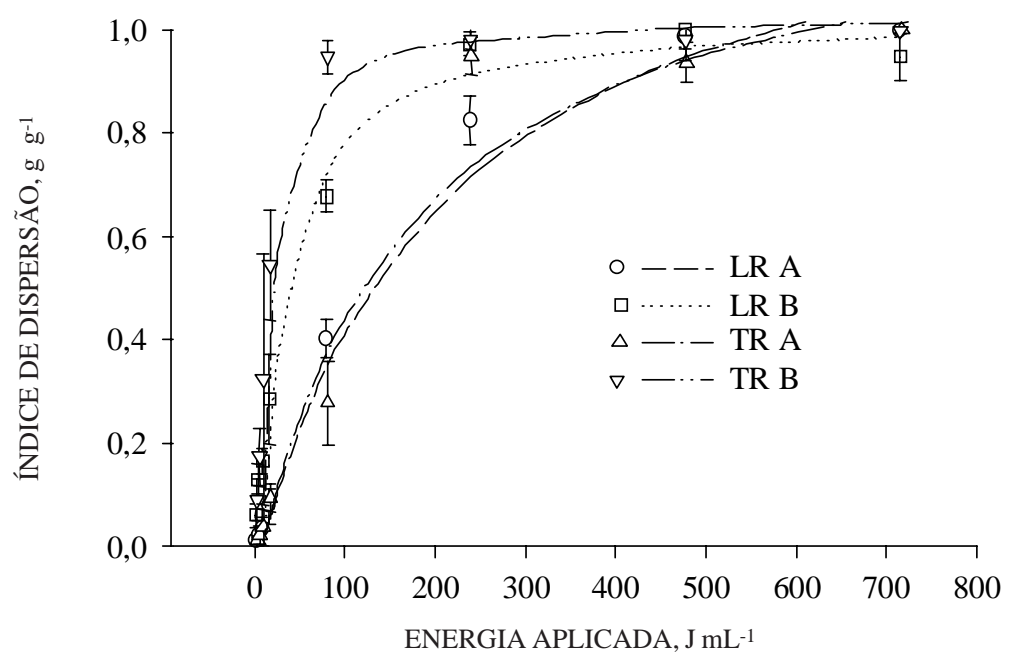

Figura 1. Curvas de dispersão para os horizontes A e B do Latossolo Roxo e da Terra Roxa Estruturada (dados normalizados, quatro repetições). 
ajustados para o horizonteA (Quadro 3) apresentavam valores de índice de dispersão normalizados acima de um a altos níveis de energia, considerou-se como energia para máxima dispersão aquela cujo índice atingisse o valor 1 . As curvas dos horizontes A apresentaram comportamento semel hante.

Utilizando equações ajustadas para dados não normalizados (Quadro 3), cal cularam-se os níveis de energia necessários à obtenção dos valores deíndice de dispersão observados em análise granulométrica (6.000 rpm durante $20 \mathrm{~min}$ ) com e sem dispersante (Quadro 4). Observa-se que, para o primeiro caso, no Latossolo Roxo, em ambos os horizontes, a energia equivalente foi a mesma, ou seja, $463 \mathrm{~J} \mathrm{mL-1,}$ enquanto, para a Terra Roxa Estruturada, os valores foram diferentes entre os dois horizontes.
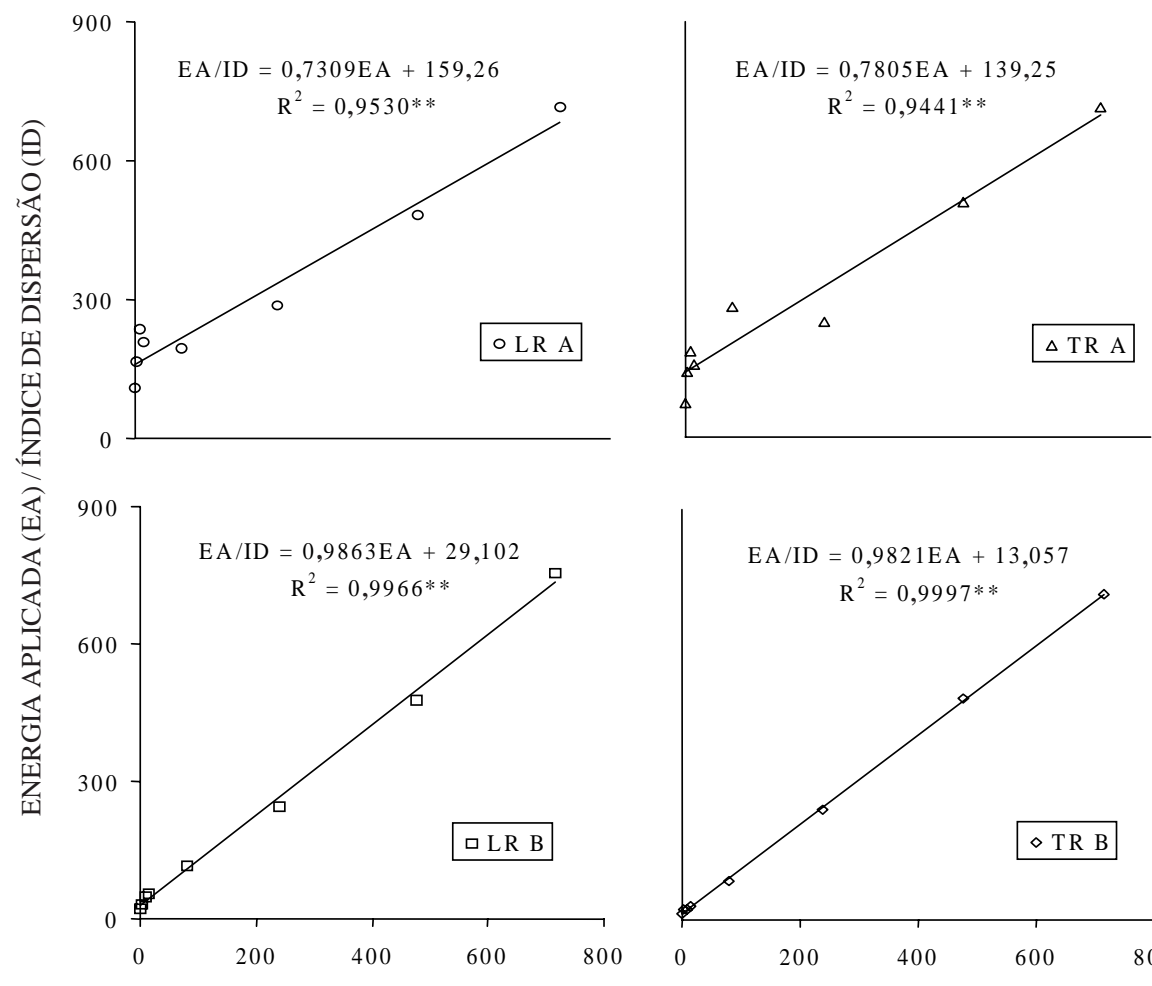

ENERGIA APLICADA (EA), $\mathrm{J} \mathrm{mL}^{-1}$

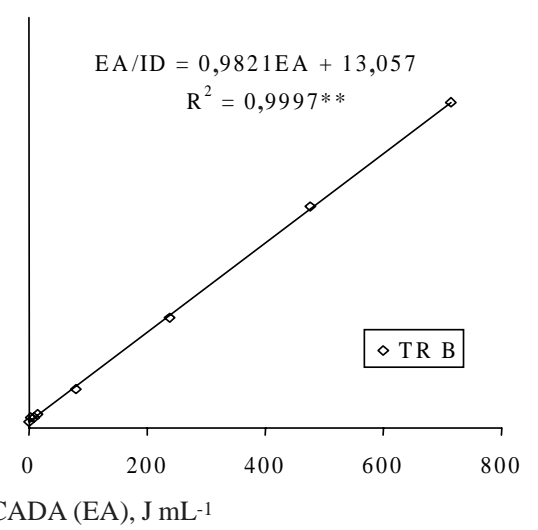

Figura 2. Curvas de dispersão linearizadas para os horizontes A e B do Latossolo Roxo e da Terra Roxa Estruturada (dados normalizados).

Quadro 3. Equações ajustadas e valores do índice de desagregação (b/a) para os horizontes A e B do Latossolo Roxo e da Terra Roxa Estruturada

\begin{tabular}{|c|c|c|c|}
\hline Solo & Horizonte & E quação & b/a $\left(m L J^{-1}\right)$ \\
\hline \multicolumn{4}{|c|}{ Dados não normalizados } \\
\hline LR & $\begin{array}{l}\text { A } \\
B\end{array}$ & $\begin{array}{l}I^{(1)}=E A^{(2)} /[178,44+0,8188 E A] \\
I D=E A /[32,252+1,0931 E A]\end{array}$ & $\begin{array}{l}0,005 \\
0,034\end{array}$ \\
\hline $\mathrm{TR}$ & $\begin{array}{l}\text { A } \\
B\end{array}$ & $\begin{array}{l}\text { ID }=E A /[157,89+0,8851 E A] \\
I D=E A /[14,118+1,0620 E A]\end{array}$ & $\begin{array}{l}0,006 \\
0,075\end{array}$ \\
\hline \multicolumn{4}{|c|}{ Dados normalizados } \\
\hline LR & $\begin{array}{l}\text { A } \\
B\end{array}$ & $\begin{array}{l}I^{(1)}=E^{(2)} /[159,26+0,7309 E A] \\
I D=E A /[29,102+0,9863 E A]\end{array}$ & $\begin{array}{l}0,005 \\
0,034\end{array}$ \\
\hline $\mathrm{TR}$ & $\begin{array}{l}A \\
B\end{array}$ & $\begin{array}{l}\text { ID }=E A /[139,25+0,7805 E A] \\
\text { ID }=E A /[13,057+0,9821 E A]\end{array}$ & $\begin{array}{l}0,006 \\
0,075\end{array}$ \\
\hline
\end{tabular}

(1) Índice de dispersão; (2) Energia aplicada. 
Quadro 4. Índices de dispersão (ID) obtidos por meio de agitação mecânica com (1) e sem (2) dispersante químico pela agitação mecânica e equivalente energia ultra-sônica

\begin{tabular}{|c|c|c|c|c|}
\hline Solo & $\mid D 1^{(1)}$ & Energia & $I D 2^{(2)}$ & Energia \\
\hline & $\mathrm{C} / \mathrm{NaOH} 1 \mathrm{~mol} \mathrm{~L}^{-1}$ & $1 \mathrm{~J} \mathrm{~mL}-1$ & $\mathrm{~S} / \mathrm{NaOH} 1 \mathrm{~mol} \mathrm{~L}^{-1}$ & $\mathrm{~J} \mathrm{mL^{-1 }}$ \\
\hline $\begin{array}{l}\text { LR A } \\
\text { LR B }\end{array}$ & $\begin{array}{l}0,83 \\
0,86\end{array}$ & $\begin{array}{l}463 \\
463\end{array}$ & $\begin{array}{l}0,19 \\
0,22\end{array}$ & $\begin{array}{r}40 \\
9\end{array}$ \\
\hline $\begin{array}{l}\text { TR A } \\
\text { TR B }\end{array}$ & $\begin{array}{l}0,76 \\
0,89\end{array}$ & $\begin{array}{l}367 \\
230\end{array}$ & $\begin{array}{l}0,49 \\
0,73\end{array}$ & $\begin{array}{r}137 \\
46\end{array}$ \\
\hline
\end{tabular}

(1) ID1 = silte + argila em $\mathrm{NaOH}$. ${ }^{(2)}$ ID2 = silte + argila em água.

No caso de dispersão em água, os valores de energia foram diferentes entre todos os horizontes de ambos os sol os, mostrando não haver uma relação entre a agitação mecânica e a energia ultra-sônica.

Para um mesmo nível desta energia e, principalmente, em baixos níveis, a dispersão mostrou-se dependente das características de cada amostra de solo, tornando promissor o uso de ultrasom em estudos de estabilidade de agregados, concordando com Raine \& So (1993).

O quadro 3 apresenta o índice de desagregação (b/a) para cada horizonte. Este índice expressa a susceptibilidade do solo à desagregação por ultrasom, e foi o mesmo para dados normalizados e não normalizados, sendo seu valor tão menor quanto mais estáveis os agregados do solo. Menores valores, ou seja, maior estabilidade foi encontrada para os horizontes A de ambos os solos, os quais foram bastante próximos, enquanto a menor estabilidade foi verificada para o horizonte B da Terra Roxa Estruturada, ficando o horizonte B do Latossolo Roxo em posição intermediária.

Tais resultados concordam com os teores de carbono orgânico (Quadro 2) que variaram substancialmente entre os horizontes e diminuíram na sequência LRA > TRA > LRB >TRB, sendo a maior estabilidade de agregados relacionada com maiores teores de matéria orgânica, concordando com Tisdall \& Oades (1982); Roth et al. (1991); Faccin (1995); Fuller et al. (1995); Campos et al. (1995). Entretanto, para o horizonte A da Terra Roxa Estruturada, al tos teores de cál cio (Quadro 2) podem estar também influenciando a estabilidade de agregados (Roth et al., 1991; Reichert et al., 1993), uma vez que esta apresentou teor de matéria orgânica mais baixo que o Latossolo Roxo.

A mineralogia gibbsítica do L atossolo Roxo expressa pela maior relação $\mathrm{Gb} /(\mathrm{Gb}+\mathrm{Ct})$ e o maior teor de matéria orgânica (Quadro 2), ajudam a explicar a maior estabi-lidade deagregados do horizonteB nesse solo.
Outro aspecto a ser observado é que este índice expressa o comportamento da curva de dispersão, ou seja, a estabilidade de agregados tanto em baixos quanto em altos níveis de energia aplicados, o que poderepresentar melhor o potencial dedesagregação pelo manejo do solo, em vez de um índice que leve em conta apenas altos níveis de energia.

Esse índice, além de fornecer um valor que representa a estabilidade de agregados do solo com base em um parâmetro de energia, permite também comparar diferentes trabal hos realizados com sol os de condições diferentes, desde que sejam utilizados os mesmos procedimentos, ou seja, mesma relação solo-suspensão e energia aplicada à suspensão, como observado por Edwards \& Bremner (1967); Saly (1967); N orth (1976); Raine \& So (1994).

\section{CONCLUSÕES}

1. Para os sol os estudados, não há relação entre a agitação mecânica e a dispersão ultra-sônica.

2. As curvas de dispersão foram específicas a cada tipo de sol o e horizonte estudados.

3. O índice dedesagregaçãoexpressa a susceptibilidade do solo em ser desagregado pelo ultra-som.

4. Para os sol os estudados, oíndice de desagregação apresentou tendência inversamente proporcional ao teor de matéria orgânica e de gibbsita.

5. Em baixos níveis de energia, a dispersão mostrou-se dependente dos atributos de cada amostra de solo.

\section{LITE RATURA CITADA}

BLAKE, G.R. \& HARTGE, K.H. Particledensity. In: KLUTE, A., ed. Methods of soil analysis. 2.ed. Madison, American Society of Agronomy, 1986a. v.1, p.377-82.

BLAKE, G.R. \& HARTGE, K.H. Bulk density. In: KLUTE, A., ed. Methods of soil analysis. 2.ed. Madison, American Society of Agronomy, 1986b. v.1. p.363-375.

CAMPOS, B.C.; REINERT, D.J .; NICOLODI, R.; RUEDELL, J . \& PETRERE, C. Estabilidade estrutural de um Latossolo Vermelho-Escuro distrófico após sete anos de rotação de culturas e sistemas de manejo de solo. R. Bras. Ci. Solo, 19:121-126, 1995.

CAROLINO DE SÁ, M.A. Quantificação da energia para dispersão dos agregados de Latossolo Roxo e Terra Roxa Estruturada. Lavras, UniversidadeF ederal de Lavras, 1998. 92p. (Tese de Mestrado)

CHRISTENSEN, B.T. Carbon and nitrogen in particle size fractions isolated from Danish Arable soils by ultrasonic dispersion and gravity-sedimentation. Acta Agric. Scand., 35:175-187, 1985. 
CHRISTENSEN, B.T. Straw incorporation and soil organic matter in macro-aggregates and particle size separates. J . Soil Sci., 37:125-135, 1986.

CHRISTENSEN, B.T. Decomposability of organic matter in particle size fractions from field soils with straw incorporation. Soil Biol. Biochem., 19:429-435, 1987.

CHRISTENSEN, B.T. \& SORENSEN, L.H. The distribution of native and labelled carbon between soil particle size fractions isolated from long-term incubation experiments. J . Soil Sci., 36:219-229, 1985.

DAY, P.R. Particle fractionation and particle-size analysis. In: BLACK, C.A., ed. Methods of soil analysis. Madison, American Society of Agronomy, 1965. v.1. p.545-566.

DERPSCH, R.; ROTH, C.H.; SIDIRAS, N. \& KÖPKE, U. Controle da erosão no Paraná, Brasil: sistemas de cobertura do solo, plantio direto e preparo conservacionista do solo. Eschborn, GTZ-IAPAR, 1991. 260p.

EDWARDS, A.P. \& BREMNER, J .M. Dispersion of soil particles by sonic vibration. J . Soil Sci., 18:47-63, 1967.

ELTZ, F.L.F.; PEIXOTO, R.T.G. \& J ASTER, F. Efeitos desistemas de preparo do solo nas propriedades físicas e químicas de um Latossolo Bruno Álico. R. Bras. Ci. Solo, 13:259-267, 1989.

EMPRESA BRASILEIRA DE PESQUISA AGROPECUÁRIA EMBRAPA. Serviço Nacional de Levantamento e Conservação de Solo. Manual de métodos de análise de solo. Rio de J aneiro, Ministério da Agricultura, 1979. Não paginado.

ERIKSEN, J .; LEFROY, R.D.B. \& BLAIR, G.J . Physical protection of soil organic's studied using acetylacetone extraction at various intensities of ultrasonic dispersion. Soil Biol. Biochem., 27:1005-1010, 1995.

FACCIN, O.P. Influência das características químicas, físicas e mineralógicas sobre a estabilidade de agregados de diferentes grupamentos de solos. Lavras, Universidade Federal de Lavras, 1995. 67p. (Tese de Mestrado)

FULLER, L.G. \& GOH, T.G. Stability-energy relationships and their application to aggregation studies. Can. J . Soil Sci., 72:453-466, 1992.

FULLER, L.G.; GOH, T.G. \& OSCARSON, D.W. Cultivation effects on dispersive clay of soil aggregates. Can.J . Soil Sci., 75:101107, 1995.

GENRICH, D.A. \& BREMNER, J.M. A reeavaluation of the ultrasonic vibration method of dispersing soils. Soil Sci. Soc. Am. Proc., 36:944-947, 1972.

GREGORICH, E.G.; KACHANOSKI, R.G. \& VORONEY, R.P. Ultrasonic Dispersion of aggregates: Distribution of organic matter in size fractions. Can. J. Soil Sci., 68:395-403, 1988.
GREGORICH, E.G.; KACHANOSKI, R.G. \& VORONEY, R.P. Carbon mineralization in Soil size fractions after various amounts of aggregate disruption. J . Soil Sci., 40:649-659, 1989.

IGWE, C.A.; AKAMIGBO, F.O.R. \& MBAGWU, J .S.C. Physical properties of soils of southeastern Nigeria and the role of some aggregating agents in their stability. Soil Sci., 160:431441, 1995.

KLUG, H.P. \& ALEXANDER, L.E. X-ray diffraction procedures for polycrystaline and amorphous materials. New York, J . Wiley \& Sons, 1974. 716p.

KOENIGS, F.F.R. Comments on the paper by P.F . N orth (1976). J. Soil Sci., 29:117-124, 1978.

MARQUES, J J. J.G.S.M. Estimativas e comparações dos fatores erosividade das chuvas e erodibilidade de solos com horizonte B textural no Brasil. Lavras, Universidade Federal de Lavras, 1996. 119p. (Tese de Mestrado)

NORTH, P.F. Towards an absolute measurement of soil structural stability using ultrasound. J . Soil Sci., 27:451-459, 1976.

NORTH, P.F. Assessment of the ultrasonic method of determining soil structural stability in relation to soil management properties. J . Soil Sci., 30:463-472, 1979.

RAINE, S.R. \& SO, B. An energy based parameter for the assessment of aggregate bond energy. J . Soil Sci., 44:249259, 1993.

RAINE, S.R. \& SO, B. Ultrasonic dispersion of soil in water: The effect of suspension properties on energy dissipation and soil dispersion. Aust. J . Soil Res., 32:1157-1174, 1994.

REICHERT, J .M.; VEIGA, M. \& CABEDA, M.S.V. Índices de estabilidade de agregados e suas relações com características e parâmetros de solo. R. Bras. Ci. Solo, 17:283-290, 1993.

ROTH, C.H.; CASTRO FILHO, C. \& MEDEIROS, G.B. Análise de fatores físicos e químicos relacionados com a agregação de um Latossolo Roxo distrófico. R. Bras. Ci. Solo, 15:241248, 1991.

SALY, R. U se of ultrasonic vibration for dispersing soil samples. Sov. Soil Sci., 11:1547-1559, 1967.

SIDIRAS, N.; ROTH, C.H. \& FARIAS, G.S. Efeito da intensidade de chuva na desagregação por impacto de gotas em três sistemas de preparo de solo. R. Bras. Ci. Solo, 8:251-254, 1984.

TISDALL, J .M. \& OADES, J .M. Organic matter and water-stable aggregates in soils. J . Soil Sci., 33:141-163, 1982.

VEIGA, M.; CABEDA, M.S.V.\& REICHERT, J .M. Erodibilidade em entressulcos de solos do Rio Grande do Sul. R. Bras. Ci. Solo, 17:121-128, 1993.

WATSON, J .R. UItrasonic vibration as a method of soil dispersion. Soil Fertil., 34:127-134, 1971. 